\title{
A BIOQUÍMICA E A APRENDIZAGEM BASEADA EM PROBLEMAS
}

\author{
(Biochemistry and Problem Based Learning)
}

\begin{abstract}
Autores: Lúcia Helena Mendonça Vargas
Afiliação: Departamento de Bioquímica da Universidade Estadual de Londrina, $\operatorname{Pr}$
\end{abstract}

\author{
vargas@inbrapenet.com.br
}

\begin{abstract}
The traditional curricula of medical schools have emphasized the acquisition of knowledge rather than its use, in the presentation of the basic sciences. Paradoxically, great part of the advances of the medical practice have been driven by the advances in the basic sciences. The problem-based learning (PBL) which rely on principles of cognitive psychology allow a better integration of the concepts of the basic sciences and clinical practice. This article approach the pedagogical model applied in the Medical School at "Universidade Estadual de Londrina" since 1998 and how the biochemistry contents have been introduced, in specific the Metabolism block.
\end{abstract}

\section{Introdução}

Na década de 20, as mudanças propostas por Flexner e seus colegas para as escolas médicas dos Estados Unidos levaram a um crescimento e fortalecimento dos departamentos de ciências básicas. Ao mesmo tempo, a força e independência dos departamentos de ciências básicas e o surgimento de inúmeras especialidades clínicas, contribuíram para a fragmentação e duplicação dos currículos (Sweeney, 1999).

Nos últimos anos, os currículos vêm sofrendo a pressão da explosão do conhecimento científico e de seus meios de divulgação que resultam em uma sobrecarga do cognitivo e pulverização dos conhecimentos. A maioria dos currículos atuais são fragmentados em disciplinas isoladas, desvinculadas da realidade profissional, hipertrofiadas em conteúdos, voltadas para formação tecnicista e para especialidade, não formando profissionais que atendam as necessidades de saúde da sociedade.

As disciplinas de bioquímica nos currículos tradicionais apesar de serem apresentadas com coerência e organização, normalmente são definidas pelos estudantes como uma coleção de estruturas químicas e reações, dificilmente assimiladas e desintegrada da sua prática profissional. A motivação ocorre em situações onde os estudantes são levados a integrar o conteúdo de bioquímica com sua futura realidade profissional.

O método da aprendizagem baseada em problemas apresenta-se neste cenário tendo como um dos fundamentos principais capacitar o aluno a "aprender a aprender". Este método já consolidado em escolas médicas internacionais como na Universidade de McMaster, Canadá e na Universidade de Limburg em Maastricht, Holanda; está sendo implantado no curso de Medicina da Universidade Estadual de Londrina (UEL) - Paraná.

O objetivo deste artigo é apresentar brevemente o método de aprendizagem baseada em problemas e sua implantação no curso de medicina da UEL e explorar o conteúdo de bioquímica no módulo de metabolismo. 


\section{Breve Histórico}

A aprendizagem baseada em problemas - ABP (ou PBL, de problem-based learning) tem sua origem na Faculdade de Medicina da Universidade de McMaster, Canadá, nos meados da década de 60 (Camp, 1996; Komatsu, 1999).

A escola de McMaster, visando formar um médico que além de outras habilidades também tivesse a capacidade de aprendizado independente, estabeleceu que o treinamento dado aos estudantes de medicina deveria visar a educação continuada; que as aulas expositivas não seriam a principal forma de transmitir o conhecimento; o contato clínico já no início do curso e que o estudo de uma série de problemas biomédicos definiria o currículo das ciências básicas e das clínicas (Sweeney, 1999).

Logo depois, três outras escolas médicas: a Universidade de Limburg em Maastricht na Holanda, a Universidade de Newcastle na Austrália, e a Universidade do Novo México nos Estados Unidos adotaram o modelo PBL.

A partir destas quatro instituições espalhou-se um importante movimento educacional e de acordo com Norman \& Schmidt (1993) mais de 60 escolas ou universidades espalhadas pelo mundo adotaram esta metodologia.

No Brasil, as primeiras instituições a implantar o método foram a Faculdade de Medicina de Marília em 1997 (Komatsu, 1999) e o curso de Medicina da Universidade Estadual de Londrina (UEL) em 1998 (Colegiado do Curso de Medicina, 1998).

\section{Fundamentos para a Aprendizagem Baseada em Problemas (ABP ou PBL)}

O PBL como filosofia educacional aproxima-se particularmente do construtivismo, que considera que o conhecimento não é absoluto, mas construído a partir do conhecimento prévio e da visão de mundo de cada indivíduo (Camp, 1996).

Os estudos da psicologia do aprendizado do adulto têm sugerido que estes são mais motivados a aprender quando participam ativamente na incorporação do conhecimento, utilizam experiências prévias e quando o foco desta aprendizagem pode ser aplicado em situações reais (Colegiado do Curso de Medicina, 1998; Kaufman, 1998) .

De acordo com Schmidt apud Kaufman (1998) o PBL está fundamentado em três princípios da psicologia cognitiva, que são:

1. O PBL ativa o conhecimento prévio do estudante, já que os estudantes devem valer-se do conhecimento para direcionar o problema proposto. O conhecimento prévio pode ser o determinante mais importante da natureza e da quantidade de informação nova que pode ser processada.

2. Quando os estudantes discutem o caso eles trabalham no conhecimento que já lhes foi apresentado inicialmente e no novo conhecimento que é acrescentado. Os estudantes criam novas associações entre conceitos e múltiplas ligações cognitivas entre os velhos e os novos conceitos. Quanto maior o número de associações que forem criadas, melhor será a capacidade de recuperação das informações da memória pelos estudantes.

3. O PBL apresenta aos estudantes problemas como ocorreriam em situações reais. O problema e a sua resolução são pistas para o estudante aprender a solucionar problemas similares que surgirão na sua prática profissional. Estas pistas são essenciais para acessar o conhecimento prévio embutido em nossa memória.

\section{O que é PBL ou ABP?}

A aprendizagem baseada em problemas é uma estratégia didática/pedagógica centrada no aluno, que tem o problema como elemento motivador e integrador do conhecimento (Colegiado do Curso de Medicina, 1998).

Os problemas são propostos com a finalidade de fazer com que o aluno estude determinados conteúdos. Embora não constitua a única prática pedagógica no $\mathrm{PBL}$, predomina para o aprendizado de conteúdos cognitivos e integração de disciplinas Berbel, 1998). 


\section{O Currículo PBL em Londrina}

O novo projeto pedagógico do curso de Medicina da UEL é fundamentado nos princípios da pedagogia interativa, de natureza democrática e pluralista, com firme eixo metodológico que prioriza a aprendizagem baseada em problemas como metodologia de ensino-aprendizagem central, tendo com principais diretrizes: estruturação modular, viabilizando a interdisciplinaridade; currículo nuclear comum a todos estudantes, proporcionando a oportunidade de vivenciar módulos e práticas eletivas, cuja função é permitir a individualização do currículo; ensino baseado na pedagogia da interação, com conteúdos das ciências básicas e clínicas desenvolvidos de forma integrada com os problemas prioritários de saúde da população; ensino centrado nas necessidades de aprendizagem dos estudantes e no professor como facilitador; contato do estudante de Medicina com as realidades de saúde e sócio-econômicas da comunidade desde o primeiro ano do curso; adoção da avaliação formativa; terminalidade do curso em seis anos.

A estrutura do currículo é formada por nove módulos em cada ano nas quatro primeiras séries, totalizando 36 módulos e nos dois últimos anos ocorre o internato médico. Optou-se por adotar como eixos orientadores do currículo o ciclo vital - concepção, nascimento, crescimento, envelhecimento e morte - e o modelo ecológico - processo saúde-doença como resultado da interação do homem com seu ambiente de vida (Campos, 1999)

A seguir, apresenta-se a grade com os módulos de ensino nas quatro primeiras séries do curso (Quadro I) (Campos, 1999).

Quadro I - Módulos educacionais das 4 séries iniciais do Curso de Medicina da UEL.

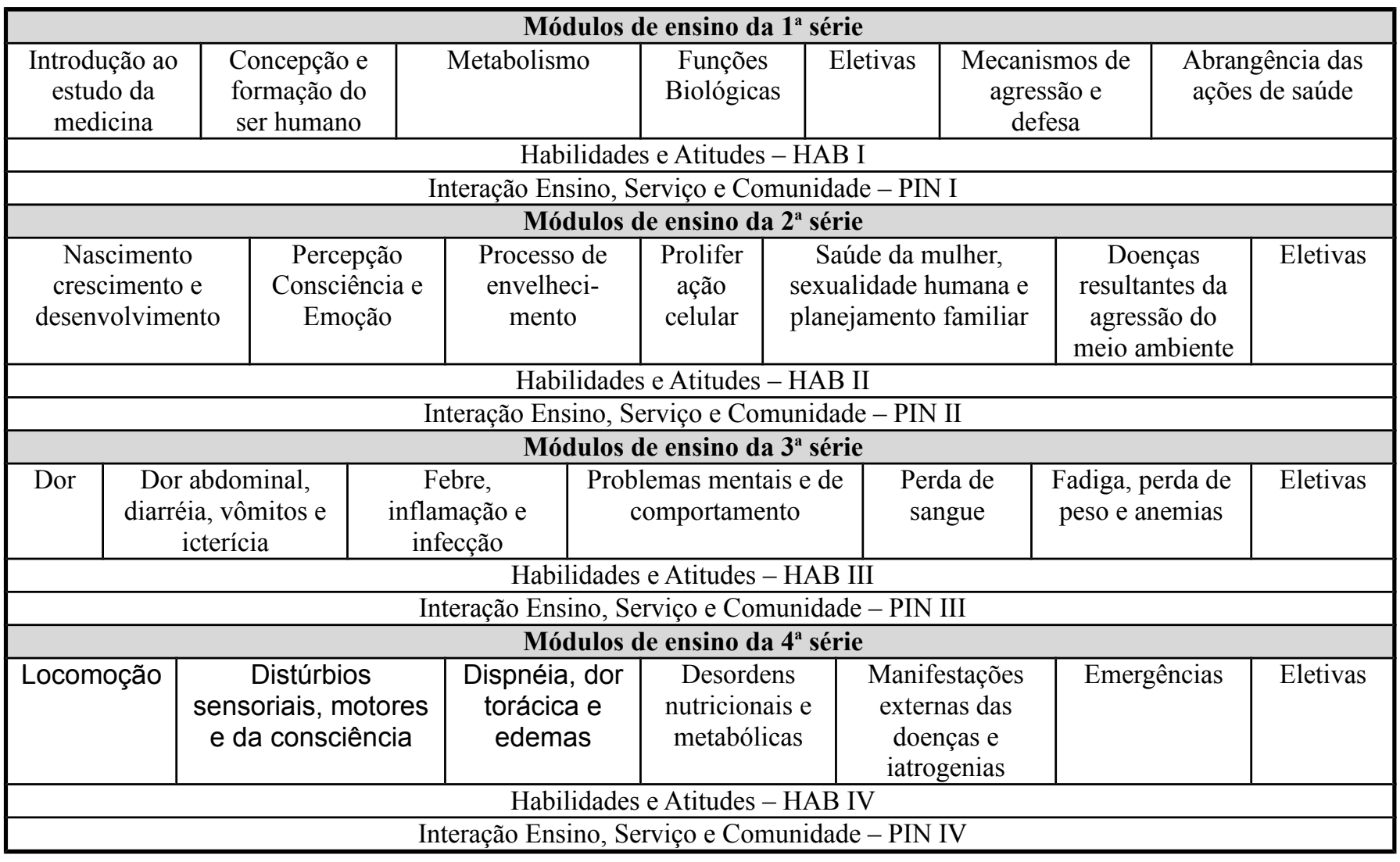

\section{Conteúdos de Bioquímica no currículo PBL}

Cada módulo temático deste novo currículo tem abordagem interdisciplinar, e o conteúdo de bioquímica está distribuído em diferentes módulos, tais como: metabolismo, funções biológicas, 
proliferação celular, desordens nutricionais e metabólicas, entre outros. Porém as proporções deste conteúdo em cada módulo são diferentes.

Considerando o grande enfoque que a bioquímica possui no módulo de metabolismo, o apresentaremos mais detalhadamente a seguir.

Para o módulo de metabolismo são utilizados conteúdos das disciplinas de anatomia, biofísica, biologia celular, bioquímica, endocrinologia, fisiologia, histologia e psicologia médica.

O módulo aborda inicialmente o processo de digestão de diferentes nutrientes incluindo os órgãos e glândulas anexas envolvidas neste processo sob os aspectos anátomo-fisiológicos. Um maior destaque é dado ao pâncreas (devido à sua função exócrina e endócrina) e ao fígado por ser o grande órgão metabólico do organismo.

As necessidades nutricionais basais, incluindo o conceito de metabolismo basal e gastos energéticos, bem como a caracterização dos nutrientes da dieta e qual as suas proporções em uma dieta equilibrada são temas relevantes neste módulo.

Para estudar o transporte transmembrana e a incorporação de nutrientes a molécula de glicose é o modelo utilizado, onde o papel dos transportadores celulares de glicose em diferentes tecidos e os processos de utilização desta glicose na ausência e presença de oxigênio e também os processos anabólicos de síntese de glicogênio e de lipídios.

A diabetes é enfocada como patologia modelo de alterações metabólicas, incorporando além do metabolismo de carboidratos, o dos ácidos graxos e o dos aminoácidos. O hormônio insulina é modelo para o estudo de síntese de proteína.

A regulação a nível celular dos processos e a nível hormonal com relação a insulina e glucagon são estudadas no módulo.

Estes temas foram transformados em dez problemas para serem discutidos nos grupos tutoriais compostos de oito estudantes e um professor/tutor. Os alunos discutem o problema seguindo a dinâmica dos "sete passos": $1^{\circ}$ Ler atentamente o problema e esclarecer termos desconhecidos; $2^{\circ}$ Identificar as questões propostas pelo enunciado; $3^{\circ}$ Oferecer explicações para estas questões com base no conhecimento prévio que o grupo tem sobre o assunto; $4^{\circ}$ Resumir estas explicações; $5^{\circ}$ Estabelecer objetivos de aprendizagem que levem o estudante ao aprofundamento e complementação destas explicações; $6^{\circ}$ Estudo individual respeitando os objetivos levantados e $7^{\circ}$ Rediscussão no grupo tutorial dos avanços de conhecimento obtidos pelo grupo (Colegiado do Curso de Medicina, 1998).

O módulo tem duração de 6 semanas, com grupos tutoriais duas vezes por semana além de atividades práticas nos diferentes departamentos envolvidos de acordo com os objetivos dos problemas e uma palestra semanal (Ribeiro et al., 2000).

Todas as atividades e problemas são elaborados por um grupo de planejamento multidisciplinar e apresentados aos estudantes através de um manual (Ribeiro et al., 2000).

Um dos problemas do módulo que busca a integração dos conteúdos de bioquímica e a clínica, cujo título é "Curiosidades de Sara", está apresentado a seguir:

"Sara, estudante de medicina, saía de um plantão enquanto tentava entender os distúrbios metabólicos observados no último paciente que atendera. Era um diabético de 13 anos que interrompera por conta própria suas injeções diárias de insulina e apresentava emagrecimento, eliminação excessiva de urina, sede intensa, fraqueza e hálito semelhante ao odor de maçã madura. Seus exames mostraram altas taxas de glicose e lipídios no sangue."

De fundamental importância tem sido o processo de avaliação no novo currículo, que é parte integrante do processo de ensino aprendizagem e ocorre como um processo contínuo. Ele proporciona aperfeiçoamento contínuo do tutor e a tomada de decisões quanto à evolução e direcionamento necessários para o aluno e para o programa.

Em cada grupo tutorial é realizada pelos alunos e tutores uma avaliação do problema estudado, bem como avaliação do desempenho do aluno quanto a conhecimentos, atitudes e habilidades, utilizandose instrumentos de avaliação formativa como auto-avaliação, avaliação interpares, avaliação pelo tutor. Ao final do módulo os alunos são avaliados em seus conhecimentos, através de uma avaliação somativa.

O módulo é avaliado pelos alunos em relação à organização, conteúdo, sistema de avaliação, recursos humanos e materiais. O desempenho do professor/tutor quanto a atitudes e habilidades nos grupos tutoriais é também avaliado pelos alunos. 
O grupo de planejamento do módulo de metabolismo vem realizando algumas alterações com relação a palestras, atividades práticas e problemas visando sempre melhor atender os objetivos do módulo. Os resultados mostraram que as mudanças realizadas de 1998 para 1999, baseadas nas avaliações do ano de 1998, tiveram um efeito positivo para os alunos e conseqüentemente na avaliação do próprio módulo em 1999.

\section{Considerações finais}

Os estudos de Norman \& Schmidt (1992) mostraram que os estudantes do PBL comparados com os do tradicional, apresentam uma maior retenção do conhecimento; uma maior capacidade para a integração de conhecimentos básicos e clínicos; melhor transferência de conceitos aprendidos para problemas novos; também são mais motivados para o aprendizado e com maior capacidade para auto-aprendizado. Também, os estudantes de PBL consideraram o ambiente de aprendizagem mais estimulante e mais humano.

Apesar do pouco tempo para avaliar a eficácia do método na UEL, algumas mudanças já são bastante visíveis particularmente a atitude mais crítica e ativa dos alunos, bem como uma visão mais holística do paciente no seu contexto biopsicosocial. Também uma aproximação muito saudável entre os professores de básico e clínico, bem como entre professores e alunos.

A avaliação como um instrumento de transformação, a organização e a liderança estudantil são aspectos que estão sendo modificados no curso de Medicina da UEL com a implantação do novo currículo. Os estudantes de medicina também reconhecem a importância das suas atitudes para que o modelo de ensino trabalhado e almejado pelo colegiado de curso realmente possa dar tão certo como nas escolas médicas de outros países (Wierzchon, 1999).

Ainda não é possível afirmar que os conceitos das ciências básicas são utilizados na prática clinica pelos alunos do PBL na UEL. No módulo de metabolismo ocorreu uma boa integração da bioquímica com as situações apresentadas nos problemas.

Um dos desafios para as ciências básicas neste novo currículo é garantir que os alunos sejam capazes de usar os conceitos científicos na prática médica.

\section{Referências Bibliográficas}

1. BERBEL,N.A.N. (1998) A problematização e a aprendizagem baseada em problemas: diferentes termos ou diferentes caminhos? Interface- Comunicação, Saúde, Educação, 2(2):139-154.

2. CAMP,G. (1996) Problem-Based Learning: a paradigm shift or a passing Fad? Publicado eletronicamente na World Wide Web, http://www.med-ed-online.org (capturado em 20/07/00).

3. CAMPOS, J.J.B.de (1999) Como abrir caminhos para a transformação do ensino médico no Brasil. Revista Brasileira de Educação Médica, 23(2/3):11-20

4. COLEGIADO DO CURSO DE MEDICINA. (1998) O novo currículo do curso medicina da Universidade Estadual de Londrina. Publicado eletronicamente na World Wide Web, http://www.uel.br/ccs/pbl (capturado em 05/02/99).

5. KAUFMAN, D. M. (1998) Problem -Based Learning: Using cases to teach about how to deal with ethical problems. NCEHR, 8(2).

6. KOMATSU,R.S. (1999) Aprendizagem Baseada em Problemas: um caminho para a transformação curricular. Revista Brasileira de Educação Médica, 23(2/3):32-37.

7. NORMAM,G.\& SCHMIDT,H., (1992) The psychological basis of problem-based learning: a review of the evidence. Academic Medicine, 67(9):557-565.

8. NORMAM,G.\& SCHMIDT,H.G. (1993) Where is the learning in problem-based learning? Pedagogue, 4:1-5.

9. RIBEIRO,M.L.L.; VARGAS,L.H.M. \& DICHI,J.B. (2000) Metabolismo A - 3Mod113. Londrina: UEL. 33p.

10. SWEENEY,G. (1999) The challenge for basic science education in problem-based medical curriula. Clinical and Investigate Medicine, 22(1):15-22.

11. WIERZCHON,P.M. (1999) Como abrir caminhos para a transformação do ensino médico: Vivendo a Realidade da Mudança. Revista Brasileira de Educação Médica, 23(2/3):27-3. 